\title{
Paving the Way Towards 5G Wireless Communication Networks
}

\author{
Prof. Mohamed Slim Alouini \\ Professor, Computer, Electrical and Mathematical Science and Engineering Division \\ King Abdullah University of Science and Technology (KAUST), Saudi Arabia
}

\begin{abstract}
$5 \mathrm{G}$ wireless communication networks are expected to fulfill the demand for higher data rates, lower latency, and/or massive connectivity of a growing number of users/devices exploiting a variety of wireless applications. This envisioned rapid increase in the use of wireless services lead the wireless research community to start looking at new technologies to address problems related to the radio-frequency (RF) spectrum exhaustion. This includes the development of (i) new techniques and concepts such as massive multiple input multiple output (MIMO) systems and heterogeneous networks to improve the spectral efficiency at the link and network layers, respectively, and (ii) novel schemes to better utilize the unregulated bandwidth in particular in the upper millimeter wave, $\mathrm{THz}$, and optical portion of the spectrum. This talk will first go briefly over the vision and goals of 5G wireless communication networks. Then it presents some of these emerging enabling technologies that need to be developed to pave the way towards the successful roll-out and operation of these future wireless networks. Finally, the talk offers at the end an overview of some of the recent results in the areas of massive MIMO systems, full-duplex communication systems, and optical (Li-Fi) wireless communication systems.
\end{abstract}

